\title{
Observation of the Moon shadow with the ANTARES neutrino telescope
}

\author{
Carla DISTEFANO* \\ Laboratori Nazionali del Sud, INFN, Catania, Italy \\ E-mail: distefano_c@lns.infn.it

\section{Colas RIVIÈRE} \\ CPPM, Aix-Marseille Universitè, CNRS/IN2P3, Marseille, France \\ E-mail: rivieredin2p3.fr
}

\section{for the ANTARES Collaboration}

\begin{abstract}
ANTARES is currently the largest neutrino telescope on the Northern Hemisphere. It consists of a three-dimensional array of PMTs deployed at a depth of $2475 \mathrm{~m}$ off-shore the Toulon cost in France. Operating since May 2008 in its full configuration, it aims to detect high-energy cosmic neutrinos. In the absence of an astrophysical standard candle, a possible way to measure the angular resolution and the pointing accuracy for a neutrino telescope is to look at "Moon shadow" in the atmospheric muon flux. The analysis method and the first results of the observation of the Moon shadow with ANTARES will be presented and discussed. The data, analysed in this work, were collected between Jan 31st, 2007 and Dec 31th 2010, for a total live time of 814 days.
\end{abstract}

XXIst International Europhysics Conference on High Energy Physics

21-27 July 2011

Grenoble, Rhône Alpes France

${ }^{*}$ Speaker. 


\section{Introduction}

ANTARES operates by detecting Cherenkov light emitted by high energy secondary muons that result from neutrino interactions inside or near the instrumented volume. The Cherenkov light is detected using an array of photomultiplier tubes (PMTs), housed in pressure resistant glass spheres. They are placed in triplets along a total of twelve detector lines, which are anchored to the sea bed at a depth of 2475 meters. Each detector line contains 25 floors of triplets, spaced 14.5 meters apart. The 12 lines are placed approximately 60 meters apart [1]. The arrival time and intensity of the Cherenkov light on the PMTs are digitized into 'hits' and transmitted to shore, where events containing muons are separated from the optical backgrounds due to natural radioactive decays and bioluminescence, and stored on disk. From the timing and position information of the hits, the muon direction and position are reconstructed using a multi-stage fitting procedure, which maximizes the likelihood of the observed hit times as a function of the muon direction and position [2].

\section{Monte Carlo simulations}

A detailed simulation of the shadow effect was carried out using a modified version of Corsika [3], where we implemented the generation of the Moon position [4]. The events were weighted according to the NSU model [5]. The detector response was then simulated taking into account the local environmental parameters as the light transmission and the optical noise recorded during the data acquisition.

The Monte Carlo simulation was used to optimize the data analysis procedure. The probability to observe the shadow effect is inferred from a hypotheses test in which test statistic is defined as the $\chi^{2}$ of the two hypotheses: moon and no moon shadow. The probability density functions were simulated for the two hypotheses and used to compute the expected significance (see Fig.1).

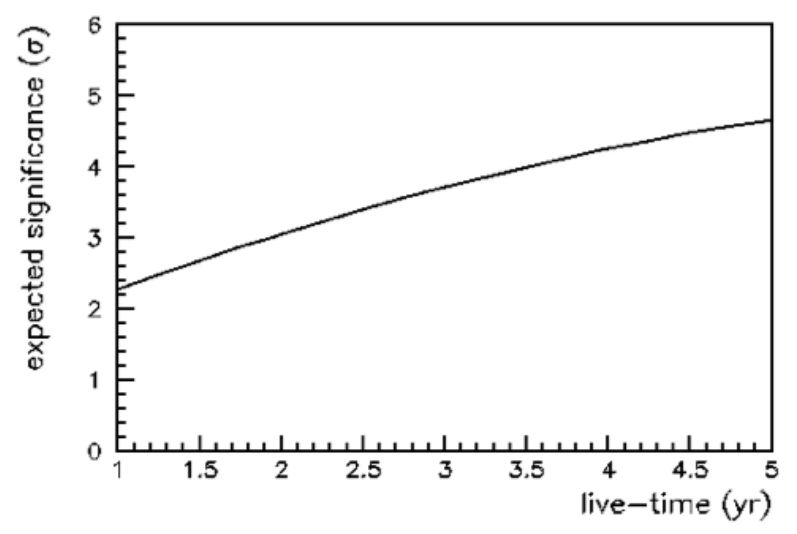

Figure 1: Expected significance for the observation of the Moon shadow effect as a function of the detector live-time. 


\section{Data analysis and present results}

After the reconstruction, the muon tracks are selected applying the following event selection criteria: the track must be reconstructed as down-going and detected when the Moon is above the Horizon. Besides, the uncertainty on the reconstructed muon track direction obtained from the fit is required to be $\leq 1^{\circ}$ and value of the log-likelihood function of the fitted muon track must be greater than a given value depending on the detector geometrical configuration, as optimized through the Monte Carlo simulations.

The event density of muons surviving the selection is plotted versus the angular distance from the Moon and fitted with a Gaussian function with a sigma of $0.4^{\circ} \pm 0.1^{\circ}$ (see Fig.2). The value of the test function for the analyzed data set is -6.2 , which represents a significance of $2.8 \sigma$ consistently with the expected one and results from alternative analysis procedures [6].

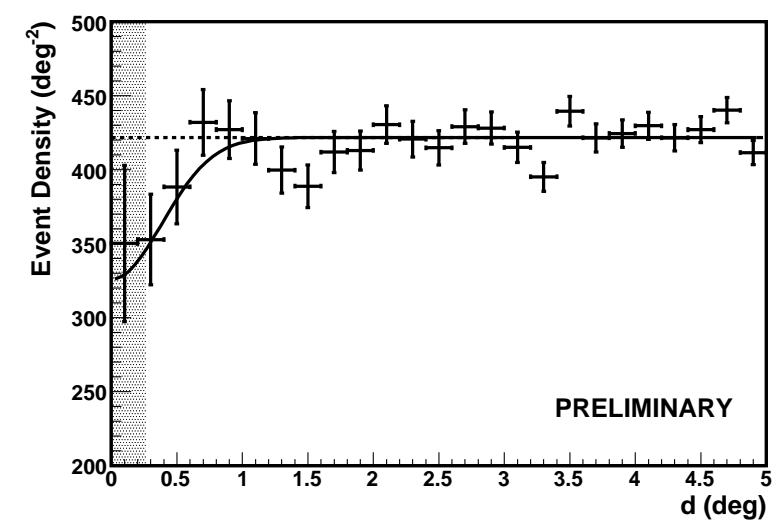

Figure 2: The event density of muons surviving the selection is plotted versus the distance $\mathrm{d}$ from the Moon (dashed area). The shadow is fitted with a Gaussian function with a sigma of $0.4^{\circ} \pm 0.1^{\circ}$.

\section{Conclusions}

A search for the observation of the Moon shadow with the ANTARES neutrino telescope has been performed on a data set corresponding to 814 days of livetime. The resulting significance of $2.8 \sigma$ is within the expected range and compatible with other analysis. Although it is not strong enough to constrain the ANTARES pointing capability, systematic studies are still ongoing aiming to optimize the event selection (track quality, number of lines, error estimate, etc.). Besides, a possible development of a track reconstruction algorithm optimized for down-going atmospheric muons together with a statistics increase should improve this results.

\section{References}

[1] M. Ageron et al., ANTARES: The first undersea neutrino telescope, NIM A 656 (2011) 11.

[2] S. Adrian-Martinez et al., First Search for Point Sources of High Energy Cosmic Neutrinos with the ANTARES Neutrino Telescope, APJ (2011), arXiv:1108.0292 [astro-ph.HE].

[3] Corsika web page: http://www-ik3.fzk.de/œheck/corsika/physics description/corsika phys.html. 
[4] C. Distefano for the ANTARES coll., On the detection of the Moon shadow with the ANTARES neutrino telescope, NIM A 626-627 (2011) S223.

[5] E.V. Bugaev et al., Atmospheric muon flux at sea level, underground, and underwater, Phys. Rev. D58 (1998) 05401.

[6] C. Rivière, C. Distefano for the ANTARES coll., Moon shadow observation with the ANTARES neutrino telescope, proc. of $32^{\text {nd }}$ ICRC, Beijing 2011. 